\title{
Análisis del proceso de innovación curricular en la Escuela de Fonoaudiología de la Universidad de Chile
}

Claudia Arancibia S. Fonoaudióloga

Magíster en Educación en Ciencias de la Salud

Departamento de Fonoaudiología, Facultad de Medicina Universidad de Chile

\section{Carmen Julia Coloma T.} Doctora en Psicología

Departamento de Fonoaudiología, Facultad de Medicina Universidad de Chile

\section{Christián Peñaloza C.} Magíster en Letras Departamento de Fonoaudiología, Facultad de Medicina Universidad de Chile
Contacto con el autor: Claudia Arancibia S. Avenida Independencia 1027

Santiago - Chile

Tel: $(56$ 2) 29786605

Correo-e: carancibia@med.uchile.cl

Recibido: $30 / 07 / 2015$ Aceptado: 03/11/2015

\section{Curricular Innovation Process of the School of Speech \& Language Pathology at University of Chile}

\section{RESUMEN}

El proceso de innovación curricular de la Facultad de Medicina de la Universidad de Chile ha implicado un desafío importante para autoridades, académicos y comunidad estudiantil. La universidad ha adoptado un modelo basado en las competencias que el estudiante debe demostrar en los distintos ámbitos de la disciplina, para dar cuenta del perfil del egreso. En este sentido, la carrera de Fonoaudiología ha construido una nueva malla curricular constituida por competencias, subcompetencias e indicadores de logro para cada uno de los dominios de formación. El rediseño curricular conlleva una renovación de las metodologías didácticas y una nueva mirada en relación con el proceso de enseñanza aprendizaje, situando al estudiante en un rol más activo. Gracias al levantamiento de demandas y necesidades formativas, se construyeron cinco dominios correspondientes a los ámbitos de realización del egresado en Fonoaudiología. En este artículo se describen y explican los elementos constituyentes del nuevo currículum, detallando ámbitos relevantes y su justificación en el plan formativo global de la Escuela de Fonoaudiología.

Palabras clave: currículum, innovación curricular, competencias, perfil de egreso, fonoaudiología.

\section{ABSTRACT}

The process of curriculum innovation at the Faculty of Medicine at the University of Chile has implied an important challenge for authorities, faculty members, and students. The university has adopted a model based on competences which students have to demonstrate in different areas of the discipline in order to accomplish the graduate profile. In this respect, the Speech \& Language Pathology undergraduate program has developed a new curriculum based on competencies, subcompetencies and learning outcomes for each domain of the academic training. The new curriculum involves renewed didactic methodologies and a new approach in the learning process. This would place the student in a more active role (learner-centredness). Five domains corresponding to the different professional areas of the Speech \& Language Pathology program were developed based on the demands and training needs. The present article describes and explains the constituent elements of the new curriculum, providing information about the areas which were emphatically assumed by the Speech \& Language Pathology Department.

Keywords: curriculum, curricular innovation, competencies, graduate profile, speech \& language pathology. 


\section{Introducción}

A partir del año 2006, la Escuela de Fonoaudiología inició un proceso de innovación curricular al igual que las demás escuelas de la Facultad de Medicina de la Universidad de Chile. Dicho proceso estuvo enmarcado en la modernización del pregrado de esta casa de estudios, que busca formar profesionales con alto sentido público, crítico y aptitud para anticipar, impulsar y liderar procesos de cambios en los diversos órdenes de la vida social (Universidad de Chile, 2011).

Este conjunto de reformas a nivel de pregrado se debieron en parte a que en la última década se han producido importantes cambios en la educación superior. En particular, las exigencias de transformaciones nacen de la necesidad de contar con una estructura formativa que dé mayor flexibilidad y continuidad académica (Pey y Chauriye, 2012). Asimismo, se ha advertido un cambio en los paradigmas de la formación profesional universitaria, que ubica al estudiante como agente activo de su aprendizaje y a los docentes, como facilitadores de su proceso educativo (Mclean y Gibbs, 2010).

Otro de los motivos que avalaron el rediseño de la malla curricular se relaciona con el proceso de Autoevaluación de la escuela con miras a su primera acreditación. Si bien la unidad se acreditó por el período máximo, una de las debilidades detectadas fue la falta de explicitación del marco disciplinar que oriente la formulación del perfil de egreso (Escuela de Fonoaudiología, 2006). Dicha debilidad fue asumida en el proceso de innovación, donde se cauteló que la construcción de la nueva propuesta formativa tuviera un mayor sustento disciplinar.
En particular, para realizar la innovación curricular de la escuela fue necesario constituir, en el año 2006, una comisión local que dirigiera el proceso y que velara porque la propuesta formativa cumpliera con la misión de la universidad y con el sello institucional. Esta comisión ha estado constituida por diferentes académicos a través del tiempo ${ }^{1}$. Finalmente, este proceso curricular se concretó en un plan de formación basado en competencias que entró en vigencia en el año 2013.

En este punto, cabe distinguir la noción de competencia para la formación profesional, asumiendo que existen diversas acepciones según los contextos en los cuales se aplique. Al respecto se construyó una conceptualización de competencia consensuado luego de un proceso amplio de reflexión en la comunidad académica, coincidiendo en que:

Una competencia es el saber actuar de manera pertinente en situaciones y contextos varios, enfrentando problemas con un claro criterio de calidad, para lo cual se articulan y movilizan recursos internos (conocimientos, experiencias, etc.), de contexto y de redes, estando en condiciones de dar razón de sus decisiones y actuaciones, y haciéndose cargo de los efectos e impactos de los mismos (Hawes, 2005, en Armanet y De Barbieri, 2009, p. 346).

La relevancia de este concepto implica que el logro de las competencias incluidas en el plan de formación le entrega al egresado el respaldo

\footnotetext{
1 La comisión ha sido dirigida sucesivamente por las académicas Carmen Julia Coloma, Claudia Arancibia, Ximena Hormazábal, Lilian Toledo y Virginia Varela.
} 
institucional para presentarse como profesional frente a la sociedad y al equipo de salud que integre (Armanet y De Barbieri, 2009).

Es así como el modelo basado en competencias se concretó en un currículum que contempló dominios o ámbitos de realización, competencias y subcompetencias que, en su totalidad, constituyen el perfil de egreso.

Los dominios se describieron como un campo de la profesión configurado por una "familia de problemas" pertinentes, que forman parte de la propuesta formativa de la carrera y, por ello, son un componente identitario de la misma (Hawes, 2010). A su vez se entendió por perfil de egreso la declaración institucional acerca de las características de sus egresados, que se expresan en términos de competencias aplicables a diferentes dominios de la acción profesional. Las competencias que constituyeron la matriz curricular se agruparon en torno a las denominadas ciencias básicas (anatomía, biología, fisiología, lingüística, entre otros) y a las competencias transversales o genéricas, comunes a grandes conjuntos de disciplinas y especialidades (Corvalán y Hawes, 2006).

La escuela definió diferentes dominios que guiaban la construcción del perfil de egreso. Se llevó a cabo un proceso de levantamiento de necesidades formativas y ámbitos de desempeño, con consulta a actores relevantes (académicos, estudiantes, egresados y empleadores), lo que permitió proponer cinco dominios: Intervención, Investigación, Promoción y Prevención, Compromiso Social, Gestión y Genérico Transversal, identificando al de Intervención como eje de la formación disciplinar.
El claustro de la Escuela de Fonoaudiología acordó intencionar una formación de pregrado que estuviera marcada por un sello de Compromiso Social, que se manifestara transversalmente en todos los cursos impartidos por la unidad. Esto llevó a la decisión de considerarlo un dominio de la formación de pregrado. Además, se consensuó que junto al de Intervención, el currículum enfatizaría la Investigación disciplinar, constituyendo esta un nuevo dominio. En consecuencia, la malla innovada se distingue por un mayor énfasis en estos tres dominios.

El énfasis en los dominios propuestos (Intervención, Compromiso Social e Investigación) concuerda ampliamente con la literatura especializada en currículum de educación superior, donde se afirma que si una institución desea implementar un proceso de reforma curricular exitoso, necesita definir su currículum nuclear o core curriculum en función de los requerimientos de la comunidad a la que sirve (Mclean y Gibbs, 2010).

El propósito de este artículo es describir y analizar los dominios antes mencionados, porque constituyen parte esencial del nuevo currículum de la Escuela de Fonoaudiología de la Universidad de Chile.

\section{Desarrollo}

El nuevo plan de formación de la Escuela de Fonoaudiología contiene la malla curricular, el perfil de egreso, el reglamento que rige la formación de pregrado y los dominios de: Intervención, Investigación, Promoción y Prevención, Compromiso Social, Gestión y Genérico Transversal (Escuela de Fonoaudiología, 2013). Cada dominio se organiza en 
torno a competencias, subcompetencias e indicadores que evidencian el logro de las competencias. La Figura 1 ilustra la organización de estos componentes formativos.

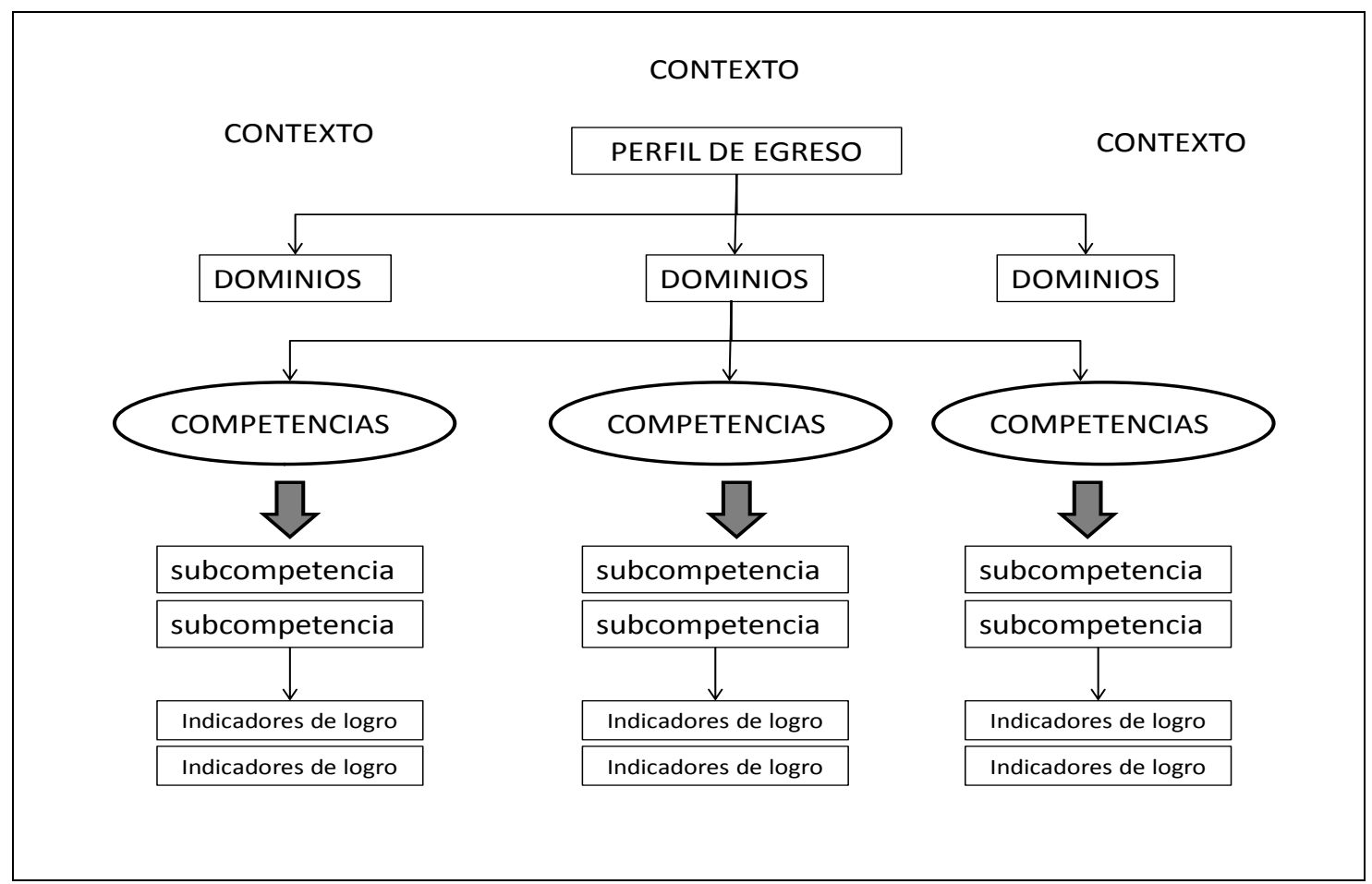

Figura 1. Operacionalización de variables

A continuación, se presentan los dominios que constituyen el sello formativo perteneciente al perfil del egresado de la Escuela de Fonoaudiología de la Universidad de Chile. Además, se analizan aquellos aspectos considerados nucleares en la formación de pregrado y que por ello aseguran su pertinencia en la malla.

Dominio de Intervención. Se define Intervención como el ámbito de acción que implica construir espacios de interacción profesional por medio de la evaluación, el diagnóstico y el tratamiento de la comunicación (lenguaje, cognición, habla y voz), motricidad orofacial, alimentación oral, audición y sistema vestibular. Lo anterior se realiza al servicio de personas y/o comunidades con necesidad de apoyo fonoaudiológico en cualquier etapa del ciclo vital. Este ámbito está en coherencia con el dominio transversal de Compromiso Social, ya que se espera un actuar integral que permita trabajar en conjunto con otros actores relevantes. Además, este actuar debe considerar el contexto, la diversidad y los derechos de las personas. Finalmente, en su desempeño profesional debe respetar las normas éticas.

\section{a) Ámbitos de Intervención}

Se redefinieron los ámbitos de Intervención ya que a las áreas tradicionales de la Fonoaudiología (habla, lenguaje, voz, audición y sistema vestibular) se incorporaron otras temáticas que se han desarrollado en el ámbito de la profesión en Chile y 
el mundo, entre las que se cuenta la motricidad orofacial, la alimentación oral y el enfoque cognitivo, que se detallan a continuación.

En primer término, la inclusión de la motricidad orofacial y la alimentación oral obedecen a las demandas de ejercicio profesional a nivel nacional e internacional. Se amplió el abordaje terapéutico, tanto en la población infantil como adulta, se abrieron nuevos campos de acción del fonoaudiólogo, posibilitando una formación más específica y dirigida a la resolución de dificultades de deglución y alimentación en la población. Las temáticas mencionadas se tradujeron en competencias y subcompetencias al interior de cursos diseñados especialmente para ello, entre los que se cuentan: "Motricidad Orofacial" e "Intervención de la Deglución y Motricidad Orofacial". Además en la práctica profesional en quinto año se aseguró que estas temáticas estuvieran presentes en, al menos, una de las áreas de desempeño profesional.

Similar situación ocurrió con el enfoque cognitivo, ya que se consideró fundamental en el dominio de Intervención el abordaje terapéutico de personas con dificultades de la comunicación que pueden ser tratadas desde una perspectiva cognitiva. Al igual que en las áreas de motricidad orofacial y de alimentación oral, se construyeron competencias y subcompetencias, las que dieron origen a cursos que abordaron específicamente esta temática, tanto en la población infantil como en la adulta. Ellos son: "Fundamentos Cognitivos de la Comunicación" e "Intervención Cognitiva Comunicativa en Adultos".

\section{b) Proceso terapéutico en Fonoaudiología}

Se trata de un proceso flexible, dinámico y sujeto a las necesidades de la persona o comunidad requirente. Incluye una competencia general en el dominio de Intervención sobre los conocimientos y las habilidades del proceso terapéutico (competencia 2 de Intervención). Ahí se explicitó que el fonoaudiólogo o fonoaudióloga de la Universidad de Chile "aplica un modelo de intervención atingente a las características y necesidades de las personas que requieren apoyo fonoaudiológico y del terapeuta" (Indicador de logro 2.2.4 de la subcompetencia 2.2 de Intervención) y se definieron con la mayor diversidad y amplitud posible los componentes de los procesos de diagnóstico y de tratamiento (subcompetencias 2.3 y 2.4 de Intervención). Finalmente, ello se concretó en el curso "Intervención fonoaudiológica y Bioética Aplicada", sin perjuicio de que en niveles superiores continúa el desarrollo de esta competencia, con temáticas e indicadores de logro más específicos y propios de cada área.

La redefinición del proceso terapéutico también contribuyó a dar respuesta a la debilidad encontrada en el proceso de acreditación, acerca de la ausencia de explicitación del marco disciplinar.

\section{c) Espacios de interacción}

Otro aspecto importante en la definición del dominio fue la idea de "construcción de espacios de interacción". Este aspecto fue discutido ampliamente por parte del claustro, ya que fue relevante situar la intervención fonoaudiológica desde una perspectiva más cercana a un modelo biopsicosocial y más lejana al biomédico, que funcionó como eje de los planes 
formativos anteriores. Esta definición implicó que el fonoaudiólogo debía ser capaz de contar con las competencias para construir relaciones de intersubjetividad, combinar, asociar y mezclar coherentemente puntos de vista, ideas, valoraciones y estrategias de acción, de acuerdo con las necesidades comunicativas de la persona a la que se acompaña (Romero, 2012).

Este aspecto también se materializó en la competencia 2 de Intervención, al explicitar que el fonoaudiólogo o fonoaudióloga: "Se caracteriza críticamente a sí mismo como sujeto comunicativo, considerando su contexto social y cultural" (Indicador de logro 2.2.1 de la subcompetencia 2.1 de Intervención); “Reconoce el impacto en sí mismo al intervenir a un sujeto" (Indicador de logro 2.6.2 de la subcompetencia 2.6 de Intervención); y "Realiza acciones destinadas a su autocuidado con el fin de enfrentar el impacto que le puede producir una intervención determinada" (Indicador de logro 2.6.4 de la subcompetencia 2.6 de Intervención); “Demuestra en su actuar terapéutico respeto por la diversidad cultural, educacional, étnica, socioeconómica, política, demográfica, religiosa y de género de las personas que requieren apoyo fonoaudiológico" (Indicador de logro 2.1.1 de la subcompetencia 2.1 de Intervención); "Establece y mantiene los vínculos necesarios con las personas que requieren apoyo fonoaudiológico y con su entorno, en beneficio del proceso de intervención y de acuerdo con las características y necesidades del sujeto" (Indicador de logro 2.2.7 de la subcompetencia 2.2 de Intervención); "Mantiene comunicación permanente respecto de la terapia, adecuándose a los destinatarios (otros profesionales, familia, el mismo sujeto, etc.) de manera oral y/o escrita" (Indicador de logro 2.4.8 de la subcompetencia 2.4 de Intervención); y "Reconoce la incertidumbre que se produce en el espacio terapéutico y es capaz de manejarla en beneficio del sujeto tratado" (Indicador de logro 2.6.6 de la subcompetencia 2.6 de Intervención).

Asimismo, es importante destacar que al interior del claustro se generó un interés por desarrollar habilidades comunicativas verbales y no verbales en la formación de pregrado, ya que estas contribuyen al desarrollo personal de los estudiantes y les permite enfrentar las dificultades propias de la disciplina, tanto en ambientes clínicos como educativos. Esto se ha traducido en un énfasis en la formación que considera mayor empoderamiento de la persona del terapeuta. En este sentido, se pretendió que el estudiante logre la reflexión, el análisis crítico y la comunicación eficaz, de tal modo que al enfrentarse al paciente dentro del proceso terapéutico lo realice con las mejores herramientas (Varela, Peñaloza, Azócar y Vega, 2014).

Todo lo anterior, junto con las demás competencias genérico-transversales definieron un conjunto complejo e integrado de características que se espera formar en el estudiante de pregrado más allá de lo estrictamente disciplinar y que instalan este espacio terapéutico intersubjetivo como sello formador del estudiante de Fonoaudiología de la Universidad de Chile.

\section{d) Actuar fonoaudiológico}

Otro aspecto importante de mencionar es que la definición del dominio de Intervención dio cabida al actuar fonoaudiológico en contextos diversos, lo cual 
demanda al estudiante mayor flexibilidad en el desempeño profesional, ya que debe adaptar sus estrategias y poner en práctica sus conocimientos al servicio de la persona o comunidad con quienes interactúa.

Una forma de ilustrar la consideración del contexto en el actuar fonoaudiológico es el abordaje de los trastornos del lenguaje infantil, donde se puede intervenir de manera diferente a un niño, dependiendo de si se trata de un contexto clínico o de uno educativo. En este caso, el o la estudiante deberá identificar los elementos contextuales que le permitan seleccionar un enfoque de intervención pertinente que responda a las necesidades de apoyo terapéutico.

\section{e) Relación con la comunidad}

Se explicitó que la relación terapéutica no solo se concibe como una relación de un fonoaudiólogo o fonoaudióloga con una persona, sino también con su entorno y familia (Indicador de logro 2.4.6 de la subcompetencia 2) o con una comunidad completa que requiera de apoyo fonoaudiológico. Lo anterior representa, en consecuencia, un desafío mayúsculo en el actual proceso de implementación, pues debe dotársele de sentido dentro del plan de formación del estudiante a los cursos de "Sociedad, Cultura y Comunicación" y de "Promoción en Salud", que apuntan justamente a trabajar con comunidades. Así, se busca promover que dichos espacios comunitarios se integren al itinerario formativo, dando sentido a la lógica de un plan que contempla competencias integradas y complejas.
Dominio de Investigación. La Escuela de Fonoaudiología definió el dominio de Investigación como el "ámbito de acción que implica generar conocimientos para aportar a la calidad de vida de las personas y contribuir al desarrollo de la disciplina, en coherencia con el dominio transversal de Compromiso Social".

En cuanto a la definición del dominio, es necesario señalar que el claustro fue enfático en declarar una clara intención que las competencias de este dominio reflejaran una lógica, en la cual se entrelazara el desarrollo disciplinar con el sello de compromiso a grupos o personas que requieran apoyo fonoaudiológico. Es así como se pretende que los estudiantes realicen una investigación desde el diseño hasta la ejecución de un proyecto, abordando el análisis de la información con juicio crítico, siempre enmarcado en principios éticos y bioéticos. Asimismo, es importante señalar que se espera que la experiencia en investigación estimule al estudiante a proseguir con su formación de posgrado y continuar aportando al desarrollo disciplinar y al conocimiento de los problemas de la comunidad.

El dominio se compone de tres competencias que son comunes a todas las carreras de la facultad. La competencia 1 se refiere al análisis crítico de la información del quehacer disciplinar. Se decidió que esta competencia fuera transversal en los primeros dos años, fundamentalmente en cursos de lingüística y en asignaturas de ciencia básica, propendiendo a que el estudiante realice búsquedas bibliográficas hasta la integración de la información recopilada en un marco teórico pertinente.

De acuerdo con la lógica de aprendizajes progresivos, se cauteló que la malla curricular 
contara con cursos específicos para la formación en investigación, correspondiente a la competencia 2 que busca que los alumnos diseñen un proyecto de investigación. Es así como se diseñaron cursos de metodología en investigación a lo largo del tercer año de la carrera.

La competencia 3 se relaciona con la ejecución del proyecto diseñado previamente. Al igual que en la competencia 2 se materializó en dos cursos de cuarto nivel, en primer y segundo semestre donde se finaliza con la elaboración de un seminario de título. Cabe señalar que a los cursos de "Proyecto de Investigación" se les asignaron créditos más altos que a las demás asignaturas de la malla, demostrando la relevancia que este dominio representa para la unidad.

Finalmente, es destacable que en forma incipiente, se ha comenzado a considerar la alfabetización académica como parte de la formación disciplinar. Específicamente, la competencia 1 del dominio de Investigación, relacionada con búsquedas bibliográficas, es aquella vinculada al desarrollo de habilidades de alfabetización académica. Esto responde a las actuales perspectivas en pedagogía universitaria en las cuales la institución se hace cargo del desarrollo de las habilidades comunicativas verbales, no verbales y escritas que se consideran propias del quehacer disciplinar y profesional.

Dominio de Compromiso Social. Se definió al dominio de Compromiso Social como aquel "ámbito de formación transversal que propende a un actuar comprometido con el bienestar de la comunidad, valora la diversidad, respeta el medioambiente y se responsabiliza éticamente por las decisiones asumidas y sus consecuencias".

A continuación se describen y analizan aquellas características del sello de compromiso social.

\section{a) Perfil de Compromiso Social}

La decisión de contar con un dominio de Compromiso Social fue el resultado de una profunda discusión dada por el claustro, frente a la necesidad de asegurar y explicitar que el egresado de Fonoaudiología sea un sujeto comprometido con el bienestar de la comunidad. Dicha decisión explica el hecho de que, desde una perspectiva estricta de competencias, este dominio del perfil de egreso no correspondió, en rigor, a un ámbito profesional de acción. Sin embargo, al proponerlo como dominio se cauteló que a lo largo del proceso formativo se mantenga el sello de compromiso social en forma coherente y permanente.

\section{b) Transversalidad del dominio}

Se pretende abarcar todo el actuar fonoaudiológico, tanto en Intervención, como en Investigación, Gestión y Promoción y Prevención en salud. Es por eso que este dominio se definió como transversal, es decir, no se forma en cursos exclusivamente creados para su desarrollo, sino que está presente a lo largo de toda la extensión del plan formativo.

Esta definición asumió que todos los componentes del plan de formación aportan explícita, responsable y coordinadamente a su consolidación. Esto implica que los distintos programas de cursos declaran contribuir al desarrollo 
de sus competencias con instancias concretas dirigidas tanto a la formación, como a la evaluación pedagógica con una visión progresiva a lo largo de los ciclos formativos.

\section{c) Énfasis del dominio}

La definición del dominio (competencias, subcompetencias e indicadores de logro) se focalizó en los siguientes aspectos: monitoreo del actuar del fonoaudiólogo o fonoaudióloga en términos éticos y legales, reflexión crítica acerca de su quehacer profesional y especial atención a los grupos de mayor vulnerabilidad en su actuar profesional. Se espera que estas tendencias contribuyan a mejorar la calidad de vida de las personas o grupos que requieren apoyo fonoaudiológico, ya sea a través de la intervención profesional, como de la investigación, la gestión o las acciones de prevención y promoción de salud.

Respecto de la subcompetencia relacionada con el ámbito ético y legal, esta se desarrolló especialmente en asignaturas clínicas del dominio de Intervención, dado que es en el actuar terapéutico donde el estudiante debe demostrar un comportamiento acorde con los principios éticos y legales, aunque también está incorporado en el dominio de Investigación. Además, esta subcompetencia se consideró en la mayoría de los cursos de intervención fonoaudiológica, ya que responde justamente a la necesidad de incorporar esta temática en el proceso terapéutico fonoaudiológico.

En términos de la valoración de la diversidad de las personas, se cauteló que en todos los cursos a cargo de la unidad, se incorporaran indicadores específicos relacionados con esta temática. Un ejemplo de ello correspondió a los cursos del área de lingüística aplicada, donde se muestran las variaciones que se producen en los distintos componentes del lenguaje con el fin de comprender, valorar y respetar la diversidad lingüística.

Por último, respecto de la atención a grupos de mayor vulnerabilidad, se procuró de forma explícita que, desde el primer año de la carrera, las diversas atenciones a personas que requieren apoyo fonoaudiológico sean dirigidas a grupos con estas características. En especial, este énfasis se manifiesta en otro de los dominios de la malla innovada denominado Promoción y Prevención, el cual contempló el desarrollo de acciones tendientes a mejorar el bienestar de la población y la prevención de problemas del ámbito fonoaudiológico.

Por otra parte, dentro del claustro y de la comisión local se originó una discusión en torno al grado de responsabilidad que puede asumir un plan de formación de pregrado respecto del futuro actuar profesional de sus egresados. Es por eso que la definición del dominio de Promoción y Prevención recurre a un verbo de deseo ("propende") como característica del egresado, asegurada por las instancias experienciales del proceso formativo, y no a un verbo de acción definido, como ocurre en los restantes dominios del perfil de egreso ("demuestra"). Del mismo modo, no es posible garantizar que el futuro profesional mantenga una actitud de compromiso social y de comportamiento ético, sino que más bien en la unidad existe la convicción de que la formación de calidad conlleva necesariamente un sello de compromiso social, acorde con la misión y visión de la Universidad de Chile. 
En este mismo sentido es importante recordar que, como ya se expuso anteriormente, el dominio de Investigación también está identificado como sello de la formación de pregrado, por lo cual la unidad ha establecido directrices claras para que la investigación en fonoaudiología enfatice y promueva la vinculación del desarrollo disciplinar con la contribución a mejorar la calidad de vida de la población.

\section{Comentarios}

En el presente artículo se ha mostrado en líneas generales el proceso de rediseño curricular al interior de la Escuela de Fonoaudiología. Se ha puesto énfasis en los dominios de Intervención, Investigación y Compromiso Social. Se han analizado los componentes de estos dominios y su justificación en el plan formativo global.

De acuerdo con el análisis realizado, el proceso de innovación curricular ha permitido una mayor integración del currículum en términos de cautelar la mayor coherencia del plan formativo, la secuencia o itinerario de logro de las competencias y la demostración por parte de los estudiantes de las acciones asociadas, definidas claramente en cada una de las asignaturas. Asimismo, la unidad ha intencionado en este proceso que se incluyan áreas emergentes de la fonoaudiología junto con aquellas más tradicionales, lo cual pretende entregar una formación de pregrado más actualizada, acorde con los requerimientos del país.

Una de las consecuencias que ha conllevado el proceso de innovación curricular es que se ha instalado una cultura de discusión y revisión permanente del currículum. Las exigencias de un cambio de esta envergadura han obligado a una revisión profunda de los fundamentos epistemológicos, pedagógicos y disciplinares y de los alcances y perspectivas sociales de la formación entregada. Lo anterior no solo da por resultado una malla innovada en pleno proceso de implementación, sino un cuerpo académico con una mayor conciencia de sus fortalezas y de los aspectos que aún debe mejorar o replantear.

También es importante destacar la idea de que paulatinamente se observan ciertos cambios favorables en cuanto a la visión de la docencia como un aspecto de suma relevancia en el quehacer académico. Ello se evidencia en un equipo docente más integrado, en un interés genuino por revisar sistemáticamente los programas y cursos, por incorporar estrategias didácticas que sitúen al estudiante en un rol más activo y por perfeccionarse en el ámbito de la docencia universitaria.

Por otra parte, si bien es cierto que se observan cambios favorables en la innovación, no se pueden obviar las dificultades que trae consigo la implementación de un proceso de esta envergadura. Actualmente, aun cuando no existen estudios formales al respecto, los alumnos perciben, por ejemplo, una mayor carga académica (relacionada con la estimación de horas no presenciales o de estudio autónomo). En este sentido, es crucial mejorar los canales de comunicación entre profesores y estudiantes, ya que muchas veces en ello radica el fracaso de un programa formativo.

De acuerdo con Palés (2006), los profesores tienen la responsabilidad de asegurar que los estudiantes tengan claros los resultados de aprendizaje, que sean capaces de adaptar sus 
experiencias formativas a sus necesidades y que sean conscientes de que han alcanzado el nivel adecuado en las diferentes competencias.

Es importante recordar que un currículum no es algo inmutable y durante todo su tiempo de vigencia debe estar sometido a una continua evaluación y revisión, debiéndose introducir los cambios que sean pertinentes siempre que sea necesario (Palés, 2006).

Finalmente, los continuos procesos de evaluación curricular darán cuenta de si la propuesta presentada se materializa coherente y pertinentemente en la formación de los estudiantes de pregrado de la carrera de Fonoaudiología de la Universidad de Chile.

\section{Referencias}

Armanet, L. y De Barbieri, Z. (2009). Proceso de innovación curricular en la Facultad de Medicina de la Universidad de Chile. Revista Hospital Clínico Universidad de Chile, 20, 345-348.

Corvalán, O. y Hawes, G. (2006). Aplicación del enfoque de competencias en la construcción curricular de la Universidad de Talca, Chile. Revista Iberoamericana de Educación, 40(1), 1-17.

Escuela de Fonoaudiología. (2006). Informe de Acuerdo de Acreditación. Documento de publicación interna. Santiago: Autor.

Escuela de Fonoaudiología. (2013). Plan de Formación. Documento de publicación interna. Santiago: Autor.

Hawes, G. (2010). Perfil de egreso. Documento interno. Departamento de Educación en Ciencias de la Salud, Facultad de Medicina, Universidad de Chile.

Mclean, M. y Gibbs, T. (2010). Twelve tips to designing and implementing a learner-centred curriculum: Prevention is better than cure. Medical Teacher, 32, 225-230.

Palés, J. L. (2006). Planificar un currículum o un programa formativo. Revista Educación Médica, 9, 59-65.
Pey, R. y Chauriye, S. (2012). Innovación curricular en las universidades del Consejo de Rectores. Reflexiones y procesos en las universidades del Consejo de Rectores. Prácticas Internacionales. Santiago: Consejo de Rectores de las Universidades Chilenas. Recuperado de http://www.consejoderectores.cl/web/pdf/PDF_libro_CRU $\mathrm{CH} . \mathrm{pdf}$

Romero, L. (2012). Desplazamiento respecto de los saberes disciplinares de la Fonoaudiología, Revista Chilena de Fonoaudiología, 11, 99-106. http://dx.doi.org/10.5354/0719-4692.2012.24520

Universidad de Chile. (2011). Modelo educativo de la Universidad de Chile. Santiago: Vicerrectoría de Asuntos Académicos.

Varela, V., Peñaloza, C., Azócar, J. y Vega, M. (2014). Habilidades comunicativas en la persona del terapeuta. Una nueva línea educativa en la formación de fonoaudiólogas y fonoaudiólogos de la Universidad de Chile. Revista Chilena de Fonoaudiología, 13, 50-59. http://dx.doi.org/10.5354/0719-4692.2014.34679 